\title{
DISCOVERY OF TWO HIGH-MAGNETIC-FIELD RADIO PULSARS
}

\author{
V. M. Kaspi ${ }^{1}$, F. Camilo ${ }^{2}$, A. G. Lyne ${ }^{2}$, R. N. Manchester ${ }^{3}$, J. F. Bell ${ }^{3}$, \\ N. D'Amico ${ }^{4}$, N. P. F. McKay ${ }^{2}$, F. Crawford ${ }^{1}$ \\ 1) Department of Physics and Center for Space Research, 70 Vassar Street, MIT, Cam- \\ bridge, MA 02139 \\ 2) University of Manchester, Jodrell Bank Observatory, Macclesfield, Cheshire, SK11 9DL, \\ UK \\ 3) Australia Telescope National Facility, CSIRO, P.O. Box 76, Epping, NSW 1710, Aus- \\ tralia \\ 4) Osservatorio Astronomico di Bologna, via Ranzani 1, 40127 Bologna and Istituto di \\ Radioastronomia del CNR, via Gobetti 101, 40129 Bologna, Italy
}

\begin{abstract}
We report the discovery of two isolated radio pulsars having the largest inferred surface dipole magnetic fields yet seen in the population: $4.1 \times 10^{13} \mathrm{G}$ and $5.5 \times 10^{13} \mathrm{G}$. These pulsars show apparently normal radio emission in a regime of magnetic field strength where some models predict no emission should occur. They have spin parameters and magnetic fields similar to those of some magnetar candidates, but exhibit very different radiative properties. This demonstrates that if the putative magnetars are indeed isolated neutron stars, their unusual attributes cannot be only a consequence of their large inferred magnetic fields.
\end{abstract}

KEYWORDS: stars: neutron, pulsars: general, pulsars: individual (PSR J1119-6127, J1814-1744)

\section{INTRODUCTION}

Recently it has been suggested (Thompson \& Duncan 1992, Kulkarni \& Frail 1993, Vasisht \& Gotthelf 1997, Kouveliotou et al. 1998) that, in addition to the radio pulsars, there exists a class of isolated rotating neutron stars with ultra-strong magnetic fields - the so-called "magnetars." The observational properties of radio pulsars and putative magnetars are very different. Known radio pulsars, whose spin periods span the range from $0.0015-8.5 \mathrm{~s}$, rarely have observable X-ray pulsations, and, in all cases, the X-ray power is much smaller than the spin-down luminosity. By contrast, the objects that have been suggested to be magnetars, namely soft gamma repeaters (SGRs) and anomalous X-ray pulsars (AXPs), exhibit pulsations with periods $5-12 \mathrm{~s}$, and high energy emission that is many orders of magnitude stronger than their spin-down luminosity (Mereghetti \& Stella 1995). Their pulsations have gone undetected at radio wavelengths. The dichotomy is thought to be a result of 
the much larger magnetic fields in magnetars, with magnetic field decay heating the neutron star to produce thermal X-ray emission (Thompson \& Duncan 1993), or thermal emission from initial cooling enhanced by the large field (Heyl \& Hernquist 1997).

Here we report the discovery of two isolated radio pulsars, PSRs J1119-6127 and J1814-1744, which have the largest inferred surface magnetic fields yet seen among radio pulsars. The results reported here will be described in more detail by Camilo et al. (2000).

\section{OBSERVATIONS AND RESULTS}

PSRs J1119-6127 and J1814-1744 were discovered as part of an ongoing survey of the Galactic Plane using the 64-m Parkes radio telescope (Lyne et al. 2000, see also D'Amico et al., these proceedings). PSR J1119-6127 has $P=0.41 \mathrm{~s}$ and period derivative $\dot{P}=4.0 \times 10^{-12}$, the largest known among radio pulsars. We have also measured an apparently stationary period second derivative, $\ddot{P}=-4 \times$ $10^{-23} \mathrm{~s}^{-1}$, making this only the third pulsar for which this has been possible through absolute pulse numbering. PSR J1814-1744 has $P=4.0$ s and $\dot{P}=7.4 \times 10^{-13}$. Spin and astrometric parameters for both pulsars are given in Table 1. From the standard equation for the dipolar surface magnetic field $B=3.2 \times 10^{19}(P \dot{P})^{1 / 2} \mathrm{G}$, we infer surface magnetic fields of $4.1 \times 10^{13} \mathrm{G}$ and $5.5 \times 10^{13} \mathrm{G}$ for PSRs J1119-6127 and J1814-1744, respectively. These are the highest magnetic field strengths yet observed among radio pulsars.

\section{DISCUSSION}

Figure 1 is a plot of $\dot{P}$ versus $P$ for the radio pulsar population, with PSRs J1119-6127 and J1814-1744 indicated. Also shown are the sources usually identified as magnetars, namely the five AXPs and two SGRs for which $P$ and $\dot{P}$ have been measured.

Most models of the radio emission physics depend on pair-production cascades above the magnetic poles and hence on the strength of the magnetic field. However, at field strengths near or above the quantum critical field, $B_{c} \equiv m_{e}^{2} c^{3} / e \hbar=4.4 \times$ $10^{13} \mathrm{G}$, the field at which the cyclotron energy is equal to the electron rest-mass energy, processes such as photon splitting may inhibit pair-producing cascades. It has therefore been argued (Baring \& Harding 1998) that a radio-loud/radio-quiet boundary can be drawn on the $P-\dot{P}$ diagram, with radio pulsars on one side, and AXPs and SGRs on the other (see Fig. 1). The existence of PSRs J1119-6127 and J1814-1744, however, demonstrates that radio emission can be produced in neutron stars with surface magnetic fields equal to or greater than $B_{c}$.

Especially noteworthy is the proximity of PSR J1814-1744 to the cluster of AXPs and SGRs at the upper right corner of Figure 1. In particular, this pulsar has a nearly identical $\dot{P}$ to the AXP 1E 2259+586 (Fahlman \& Gregory 1981, Baykal et al. 1996, Kaspi et al. 1999). The disparity in their emission properties is therefore surprising. The radio emission upper limit (Coe et al. 1994) for 1E 2259+586 implies 
Table 1: Parameters for pulsars J1119-6127 and J1814-1744.

\begin{tabular}{lll}
\hline \hline Right ascension (J2000) & $11^{\mathrm{h}} 19^{\mathrm{m}} 14^{\mathrm{s}} .2(1)$ & $18^{\mathrm{h}} 14^{\mathrm{m}} 43^{\mathrm{s}} .0(2)$ \\
Declination $(\mathrm{J} 2000)$ & $-61^{\circ} 27^{\prime} 48^{\prime \prime} .3(6)$ & $-17^{\circ} 44^{\prime} 47(23)^{\prime \prime}$ \\
Spin period, $P(\mathrm{~s})$ & $0.4076034160(1)$ & $3.975823037(1)$ \\
Period derivative, $\dot{P}$ & $4.022930(4) \times 10^{-12}$ & $7.434(4) \times 10^{-13}$ \\
Period second derivative, $\ddot{P}\left(\mathrm{~s}^{-1}\right)$ & $-4.1(3) \times 10^{-23}$ & $\ldots$ \\
Period epoch (MJD) & 51075.0 & 51075.0 \\
Dispersion measure $\left(\mathrm{cm}^{-3} \mathrm{pc}\right)$ & $713(20)$ & $834(20)$ \\
Flux density at $1374 \mathrm{MHz}, S(\mathrm{mJy})$ & $0.7(2)$ & $0.5(2)$ \\
Surface magnetic field, $B(\mathrm{Gauss})$ & $4.1 \times 10^{13}$ & $5.5 \times 10^{13}$ \\
Characteristic age, $P /(2 \dot{P})(\mathrm{kyr})$ & 1.6 & 85 \\
Spin-down luminosity $\left(\mathrm{erg} \mathrm{s}{ }^{-1}\right)$ & $2.3 \times 10^{36}$ & $4.7 \times 10^{32}$ \\
Distance, $d(\mathrm{kpc})$ & $2.4-8$ & $\sim 50$ \\
Radio luminosity at $1374 \mathrm{MHz}, S d^{2}\left(\mathrm{mJy} \mathrm{kpc}^{2}\right)$ & $\sim 20$ & $\ldots$ \\
Braking index, $n$ & $3.0(1)$ & \\
\hline
\end{tabular}

a radio luminosity at $1400 \mathrm{MHz}$ of $<0.8 \mathrm{mJy} \mathrm{kpc}^{2}$. This limit is comparable to the lowest values known for the radio pulsar population. That the radio pulse may be unobservable because of beaming cannot of course be ruled out.

The radio-loud/radio-quiet boundary line displayed in Figure 1 is more illustrative than quantitative. However, the apparently normal radio emission from PSRs J1119-6127 and J1814-1744, and the absence of radio emission from AXP 1E $2259+586$, suggests that it will be difficult to delineate any such boundary without fine model-tuning. Furthermore, Pivovaroff et al. (2000) show, from archival data, that PSR J1814-1744 must be significantly less X-ray luminous than 1E 2259+586. The similar spin parameters for these two stars, and in turn the common features between 1E 2259+586 and the other AXPs and SGRs, imply that very high inferred magnetic field strengths cannot be the primary factor governing whether an isolated neutron star is a magnetar.

PSR J1119-6127 is notably young. Only three other pulsars having ages under 2 kyr are known: the Crab pulsar $(\tau=1.3 \mathrm{kyr})$, PSR B1509-58 ( $\tau=1.6 \mathrm{kyr})$, and PSR B0540-69 $(\tau=1.7 \mathrm{kyr})$. The age of a pulsar is given by $\tau=[1-$ $\left.\left(P_{0} / P\right)^{n-1}\right](P /(n-1) \dot{P}) \simeq P / 2 \dot{P}$, where $P_{0}$ is the spin period at birth (generally assumed to be much smaller than the current spin period) and $n$ is the "braking index," defined via the relation for the spin evolution $\dot{\nu} \propto \nu^{n}$, where $\nu \equiv 1 / P$. In the standard oblique rotating vacuum dipole model, $n \equiv \nu \ddot{\nu} /(\dot{\nu})^{2}=3$. For PSR J1119-6127, the parameters listed in Table 1 imply $n=3.0 \pm 0.1$. This is the first measured braking index for a pulsar that is consistent with the usually assumed $n=3$. 


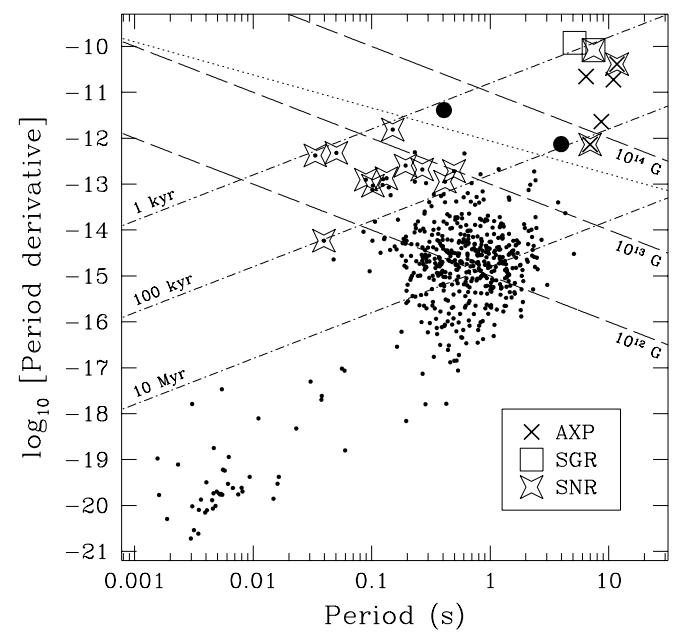

FIGURE 1. $P-\dot{P}$ diagram for radio pulsars, with SGRs and AXPs indicated. PSRs J1119-6127 and J1814-1744 are indicated with large solid circles. Lines of constant magnetic field are shown dashed, and lines of constant characteristic age $\left(\tau_{c} \equiv P / 2 \dot{P}\right)$ are shown dot-dashed. The dotted line is the proposed illustrative radio-loud/radio-quiet boundary (Baring \& Harding 1998). Radio pulsars plausibly associated with supernova remnants (SNRs) are indicated with four-point stars.

\section{REFERENCES}

Baring, M. G. \& Harding, A. K. 1998, ApJ, 507, 55

Baykal, A., Swank, J. H., Strohmayer, T. \& Stark, M. J. 1998, A\&A, 336, 173

Camilo, F., Kaspi, V. M., Lyne, A. G., Manchester, R. N., Bell, F. J., D’Amico, N., McKay, N. P.

F., Crawford, F. 2000, submitted

Coe, M. J., Jones, L. R. \& Lehto, H. 1994, MNRAS, 270, 178

Duncan, R. C. \& Thompson, C. 1992, ApJ, 392, L9

Fahlman, G. G. \& Gregory, P. C. 1981, Nature, 293, 202

Heyl, J. S. \& Hernquist, L. 1997, ApJ, 489, L67

Kaspi, V. M., Chakrabarty, D. \& Steinberger, J. S. 1999, ApJ, 525, L33

Kouveliotou, C. e. a. 1998, Nature, 393, 235

Kulkarni, S. R. \& Frail, D. A. 1993, Nature, 365, 33

Lyne, A. G., Camilo, F., Manchester, R. N., Bell, J. F., Kaspi, V. M., D’Amico, N., McKay, N. P. F., Crawford, F., Morris, D. J., Sheppard, D. C., Stairs, I. H. 2000, MNRAS, in press

Mereghetti, S. \& Stella, L. 1995, ApJ, 442, L17

Pivovaroff, M., Kaspi, V. M. \& Camilo, F. 2000, ApJ, submitted

Thompson, C. \& Duncan, R. C. 1993, ApJ, 408, 194

Vasisht, G. \& Gotthelf, E. V. 1997, ApJ, 486, L129 